\title{
Estimating the Effects of Text Genre, Image Resolution and Algorithmic Complexity needed for Sinhala Optical Character Recognition
}

\author{
Isuri Anuradha $^{\# 1}$, Chamila Liyanage ${ }^{2}$, Ruvan Weerasinghe ${ }^{3}$
}

\begin{abstract}
While optical character recognition for Latin based scripts have seen near human quality performance, the accuracy for the rounded scripts of South Asia still lags behind. Work on Sinhala OCR has mainly reported on performance on constrained classes of font faces and so been inconclusive. This paper provides a comprehensive series of experiments using conventional machine learning as well as deep learning on texts and font faces of diverse types and in diverse resolutions, in order to present a realistic estimation of the complexity of recognizing the rounded script of Sinhala. While texts of both old and contemporary books can be recognized with over $87 \%$ accuracy, those in old newspapers are much harder to recognize owing to poor print quality and resolution.
\end{abstract}

Keywords - Sinhala OCR, Optical Character Recognition, Tesseract, Deep learning.

\section{INTRODUCTION}

Optical Character Recognition (OCR) technology is designed to recognize printed texts into machine operable text. OCR is a collection of multiple steps such as scanning, preprocessing, segmentation, feature extraction, classification, recognition and post-processing. In recent literature, many OCR systems have been developed for recognizing Latin characters [1]. With the advancement of Natural Language Processing during the past few years, researchers have integrated machine learning/deep learning techniques for analysing the textual representations on digital documents. Template Matching, Neural Network (NN) and Recurrent Neural Network (RNN) are popular and widely used algorithms for character recognition. These technologies are better when applied for the other character sets, since large volumes of data are available in print media for many languages. The proposed Sinhala OCR is discussed in this paper with special focus on the text genre, image resolution and algorithmic complexities needed for training an OCR system for the Sinhala character set.

As the state of the art OCR technology, currently Tesseract is used in the training of OCR systems for many character sets. Further, Tesseract has moved from machine learning to deep learning with LSTM architecture and provides relatively better recognition competence [2]. However, algorithmic complexity

Correspondence: I. Anuradha ${ }^{\# 1}$ (E-mail isa@ucsc.cmb.ac.lk)

Received: 29-12-2020 Revised:31-05-2021 Accepted: 11-06-2021

I. Anuradha ${ }^{\# 1}$ C Liyanage ${ }^{2}, \mathrm{R}$. Weerasinghe ${ }^{3}$, are form University of Colombo School of Computing. (isa@ucsc.cmb.ac.lk)

This paper is an extended version of the paper "Deep Learning Based Sinhala Optical Character Recognition (OCR)" presented at the ICTer Conference (2020)

DOI: http://doi.org/10.4038/icter.v14i3.7231

(C) 2021 International Journal on Advances in ICT for Emerging Regions is not enough for training an OCR model, as text genre and image quality affect training a more accurate OCR model. Since large volumes of available data are in print media and they have been printed before the computer era, the documents have been printed using different techniques such as offset printing and screen printing. Therefore, common type-faces used in the history of printing should also be trained to train the model to get such text recognizable. Further, types and sizes of the fonts and size of the training text is also significant. In this paper we discuss the OCR system developed for Sinhala by estimating the effects of text genre, image resolution and algorithmic complexity.

The rest of this paper is structured as follows: Section II gives a brief overview of the related work in this area. Section III discusses some properties and characteristics of the Sinhala script as it is significant to review the complexities with regard to the particular script. Algorithmic complicacy adopted to OCR is discussed in section IV. Further, section V gives the motivation and rationale for the experimental set and systematic description on training data, word lists, and training regime adopted to develop the Sinhala OCR. Section VI presents experimental results on the OCR methods, and we also give an analysis of their performance comparison. Finally, the paper is concluded with a discussion of future works.

\section{RELATED WORKS}

Despite decades of research on the engineering aspects, the problem of Sinhala character recognition remains as a challenging issue in the OCR field. When the past few years are considered, some studies have been conducted to identify widely used font types in Sri Lanka [3]. When considering OCR for the Sinhala language, initially the K-Nearest Neighbour (KNN) algorithm-based Sinhala OCR was developed by the Language Technology Research Laboratory, University of Colombo School of Computing [3]. For the following study, commercially used font types have been employed by varying font sizes to obtain $94 \%$ of average accuracy.

Considering literature, Neural Network based Sinhala OCR systems have been developed in recent years [4], [5], [6]. In 2013, the Sri Lanka Institute of Information Technology conducted a research based on applying neural networks for Sinhala optical character recognition [4]. In this study they have only focused on 36 characters in the alphabet. Another Sinhala OCR application integrating neural networks was developed by a local research group [5]. These studies mainly focused on the character level accuracies and not on word accuracies. 
In addition, the Software Development Unit of University of Colombo School of Computing has trained a Sinhala OCR model using Tesseract 3 [7]. This system shows relatively good results only for the high-resolution images. Also, Language Technology and Research laboratory at University of Colombo is experimenting on the integration process of machine learning concepts to Sinhala OCR applications [8]. Further, Manisha et al. [9] has also tried to combine the Tesseract OCR engine with the Sinhala characters and mentions $97 \%$ of accuracy. However, the performance has not been well documented. It's well-known that Indic languages have many complexities and variations of characters which makes OCR systems hard to develop. But in the past few years, multiple studies have been conducted integrating Tesseract OCR engine for character recognition using different low resource languages such as Tamil [10], Hindi [11], Bengali [12] and Urdu [13].

\section{SINHALA SCRIPT}

The Sinhala script is an abugida or alphasyllabary script in which consonant-vowel sequences are written as units and thereby it is called a segmental writing system. The script has evolved from the Brahmi script. The letters in Sinhala are circular-shaped and are written from left to right [14]. The Sinhala script is used primarily to write the Sinhala language, which is one of the official languages of Sri Lanka spoken by about 16 million people in the country. In addition, it is also used in Sri Lanka for writing Pali, the canonical language of Theravada Buddhism, and sometimes Sanskrit, the Old IndoAryan language [15].

There are 20 vowels and 41 consonants in the Sinhala script. Since Sinhala is a segmental writing system, vowels take two representations as independent vowels: occur in the initial position of a word (infrequently occur in the middle of

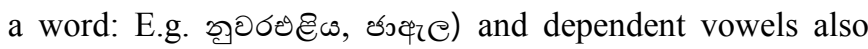
known as vowel modifiers: occur after a consonant. Figure 1 and 2 illustrate the vowels with their modifiers and consonants in Sinhala script respectively.

\begin{tabular}{|c|c|c|c|c|}
\hline \multicolumn{5}{|c|}{ Vowels and modifiers included for the training data } \\
\hline Фౌ ऐ & & נ & & $6)^{p}$ \\
\hline$\partial^{\beta}$ & & i & 60 & बढ़ \\
\hline$\check{\varphi}_{z}$ & ¿ిa & a & అ) & () \\
\hline$i^{n}$ & ¿ふаล & aว & అิ & $6\}^{p}$ \\
\hline$e^{\circ}$ & 8 & 6) & అิ૭ & ब९ \\
\hline \multirow{2}{*}{\multicolumn{5}{|c|}{ Vowels and modifiers not included for the training data }} \\
\hline & & & & \\
\hline (2) & I & (อ) 9 & 9 & \\
\hline
\end{tabular}

Fig. 1 Vowel characters and modifiers in Sinhala script

From among the vowel modifiers in figure 1, and $\circ$ (visarga) are two specific modifiers. They occur not

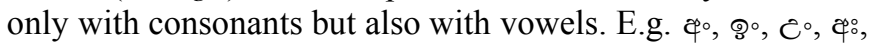
๑ి:.

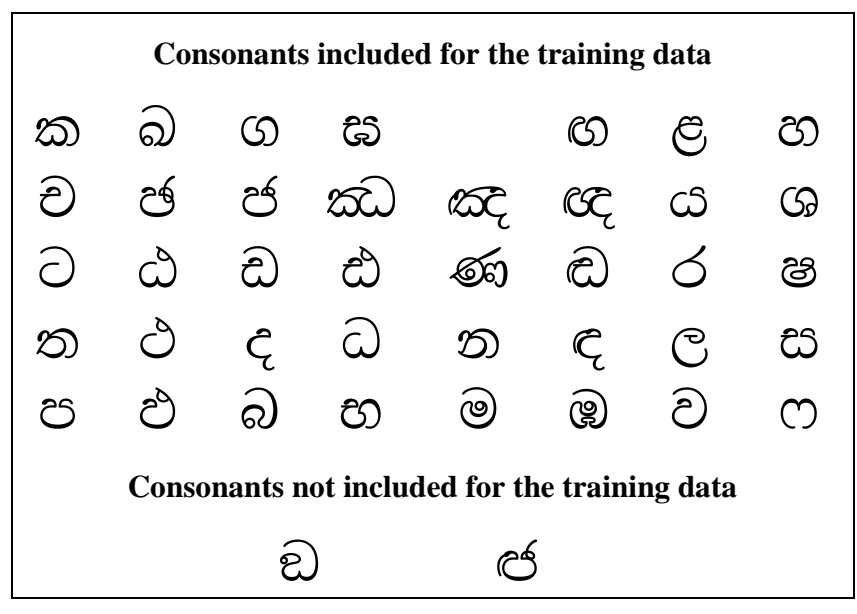

Fig. 2 Consonant characters in Sinhala script

Two vowels: 0 , oø and their corresponding vowel modifiers in figure 1 and $e \delta$ in figure 2 were not included for the training data as they do not occur in old or contemporary Sinhala books. However, $\mathbb{Q}$ in figure 2 occurs in a limited number of words in old Sinhala books. It was not included because the shape of the particular character would cause misrecognition with similar characters in Sinhala script.

Sinhala consonants imply the inherent vowel /a/ (६) when they are occur with no modifiers. Absence of the inherent vowel is marked by adding a symbol called hal lakuna or halkirima to the top of the particular consonant. E.g. $25^{p}$, อิ. Further, hal lakuna also occurs with two vowels and their modifiers. It has two shapes as illustrated in figure 3.

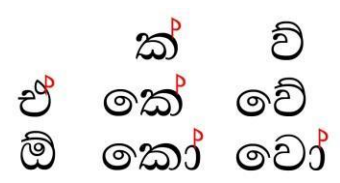

Fig. 3 Two different shapes of hal lakuna with vowels and consonants

As a segmental writing system, vowel modifiers appear above, below or to the right or left of the basic consonant. From all the consonant-vowel sequences in Sinhala script, $\theta$ is a special character as it appears as a separate symbol to represent $e+己$ sequence. As an example, following figure 4 illustrates all the consonant-vowel sequences for consonant 'هُ

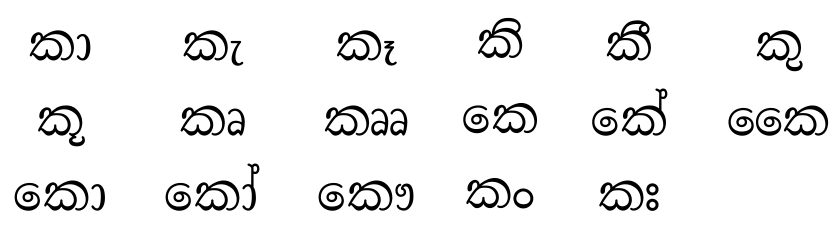

Fig. 4 Consonant 'هి' with all the vowel modifiers

There are three consonant modifiers which occur in the Sinhala script, known as rakaranshaya $(\mathrm{)}$ ), yanshaya ( s ) and rephaya $\left({ }^{\circ}\right)$. Among them rakaranshaya represents ' $\sigma$ ' (ra) and yanshaya represents ' $C S$ ' (ya) when they appear after a consonant (from which the inherent vowel has been removed). However, as symbols, rakaranshaya appears below (e.g. 200,

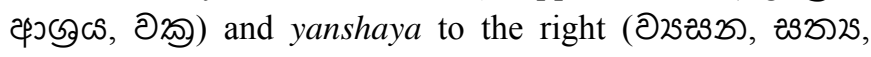


ఒॐ०ه25วอ) of the basic consonant. Further, rephaya is also used to denote ' 8 ' when it occurs before a consonant and the symbol appears on top of the basic consonant (e.g. ఏశ్రి, ఒిలి, 3ூ2 writing system while rakarakshaya and yanshaya are essential. All the vowel modifiers surround the consonant-

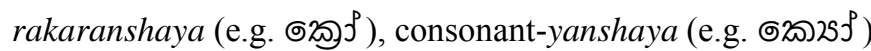
or rephaya-consonant (e.g. $\left.\sigma 2 \rho^{\rho}\right)^{p}$ ) units. Figure 5 illustrates how vowel modifiers occur with rakaranshaya.

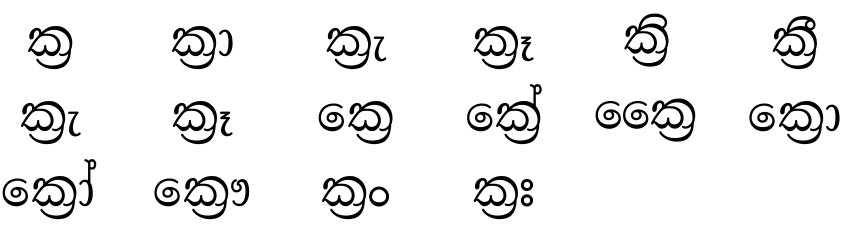

Fig. 5 Consonant ' 20 ' with rakaranshaya and the vowel modifiers

One other significant characteristic in Sinhala writing system is using compound consonants. This frequently occurred in old Sinhala books. However, in contemporary Sinhala this writing system is infrequent and therefore only the first set of compound consonants in figure 6 (which are rarely occurred in contemporary Sinhala books) have been concerned for the training data in this research.

\begin{tabular}{|c|c|}
\hline \multicolumn{2}{|c|}{$\begin{array}{l}\text { Compound consonants rarely occurred in contemporary } \\
\text { Sinhala books }\end{array}$} \\
\hline 200 & $20+$ ZWJ + 己 \\
\hline 2503 & ฉ๑ + ZWJ + ๔ి \\
\hline ๙ே) & $\omega+Z W J+œ$ \\
\hline 20 & $\ddot{\theta}+\mathrm{ZWJ}+\boldsymbol{g}$ \\
\hline 20 & $\not 3+Z W J+2$ \\
\hline 30 & $30+\mathrm{ZWJ}+\boldsymbol{g}$ \\
\hline $\mathscr{B C}$ & 2) $+\mathrm{ZWJ}+\varepsilon_{\varepsilon}$ \\
\hline 300 & $\mathfrak{B}+\mathrm{ZWJ}+\boldsymbol{\partial}$ \\
\hline 20 & (פ) $+\mathrm{ZWJ}+2$ \\
\hline$C_{s}$ & $\varepsilon_{c}+\mathrm{ZWJ}+\omega$ \\
\hline \multicolumn{2}{|c|}{ Compound consonants occurred in old books } \\
\hline (ข) & $2 \mathscr{C}_{c}+\mathrm{ZWJ}+\partial$ \\
\hline$\pi$ & $\partial+\mathrm{ZWJ}+\boldsymbol{\Phi}$ \\
\hline ๘ & $\varepsilon+Z W J+\infty$ \\
\hline อ & $\varepsilon+Z W J+\partial$ \\
\hline
\end{tabular}

Fig. 6 Compound consonants in Sinhala script

\section{SYSTEM OVERVIEW}

In our study, different Sinhala text genres were given different accuracy results. From a variety of genres, explanation and descriptive writings, narrative writings, and news reportage were selected for our purpose. When selecting documents, we considered a variety of documents and Unicode font types from different printing eras. When image resolutions were considered, low image resolutions may affect not only quality but also speed degradation of overall OCR performance, since uncertainty in character pictures produce more recognition variants. In the Tesseract engine also, high resolution images were able to give high accuracy by identifying all the punctuations, modifiers and complex letters. In the Tesseract engine, image processing is a combination of several steps such as rescaling, Binarization, Dilation / Erosion, and etc.

For the training process, we adapted and experimented on both Tesseract 3.0 (Legacy version) and Tesseract 4.0 (Deep learning) OCR engines as a tool. Tesseract has a standard level of accuracy in its engine. It's necessary to have a library file in the OCR engine called 'traineddata' which works on Sinhala inputs. This file is a concatenation of multiple files. According to the accuracy and richness in the library file, the OCR engine can work to its full potential. Sinhala language is complicated and has various types of letters including vowels, consonants, compound characters and other special types. Therefore, for Tesseract 3.0, we developed a large character set for Sinhala. It is important to mention that, for Tesseract 3.0 we need to uniquely identify each and every character. Sometimes due to the complexity of the character set, the OCR may not always detect a character correctly even if the character is included in the training files.

The preparation of data and the training process adopted for developing the Sinhala OCR model for both tesseract 3.0 and 4.0 versions are described in the following subsections.

\section{TRAINING PROCESS}

The preparation of data and the training process adopted for developing the Sinhala OCR model for both Tesseract 3.0 and 4.0 versions are described in the following subsections.

- Preparation Tesseract version 3.0

\section{A. Setting up the OCR Engine}

We installed the Tesseract version 3.0 in the Windows Operating System. Since there is no user interface of Tesseract 3.0, we used several commands in the command line to launch the application.

\section{B. Preparing training data}

The process followed by preparing training data is described below.

1) Preparing OCR alphabet for Sinhala: Initially the OCR alphabet for Sinhala was defined to collect text data. The OCR alphabet is distinct from the character alphabet which includes the basic units of training data as follows. 


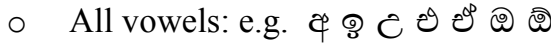

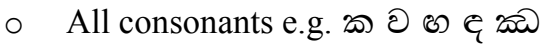

○ Consonants with touching modifiers: e.g.

○ Consonants with hal lakuna: e.g. ฉి อి

○ Compound consonants: e.g. 20 20 20

○ Compound consonants with touching modifiers: e.g.

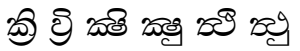

○ Non-touching modifiers: e.g. $\odot$ ஊ ஊ

○ Punctuation marks: e.g. ! ? ( )/

2) Text data collection: Text data for preparing training images were collected from the UCSC 10M Sinhala corpus and these were extracted as per the OCR alphabet that we defined. We selected 1050 words from extracted data for the preparation of training images. figure 7 shows a sample of text data.

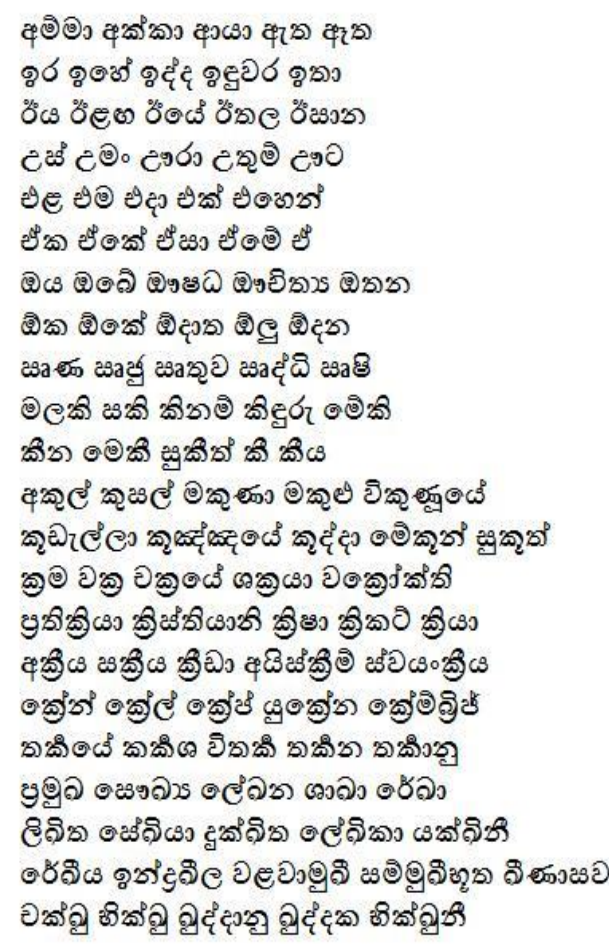

Fig. 7 Sample of text data

3) Preparing training Image: Training images were prepared for fonts widely used in Sinhala typing. The criteria followed to create text images are as follows.

Colour: grayscale

Font size of the text: $12,14,16$

DPI: 300

Fonts used: Iskoola Pota, FMAbhaya, Malithi Web, BhashithaScreen, DinaminaUniWeb

Based on the above criteria we prepared two sets of training images. The first set consisted of computergenerated images (screenshots). As an iterative process of training, the second set of training images were prepared with scanned images for the same text data. Figure 8 shows a sample of such training images.

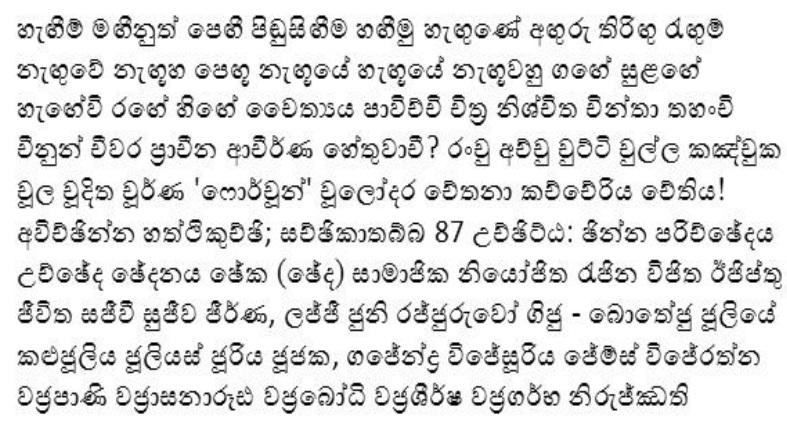

Fig. 8 Sample of training images

4) Character Segmentation: In Tesseract 3.0, character segmentation is performed using the process of creating box files. A 'box file' is a text file, which contains the necessary information of the training images. The coordinate values of the characters in the training images along with corresponding Unicode characters are stored in these box files. The segmentation of the characters in the training images was done as per the OCR alphabet we defined. Each training image should have a box file in which the number of boxes must tally with the number of training character segments in the image. Figures 9 and 10 show an image with segmented characters and a sample of box information.

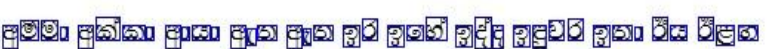

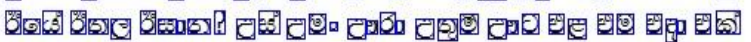

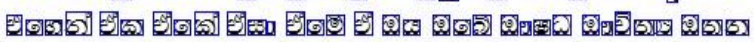

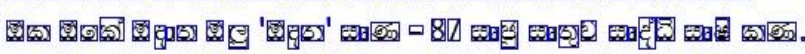

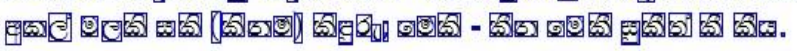

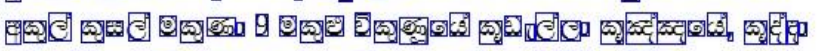

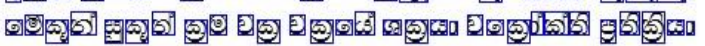

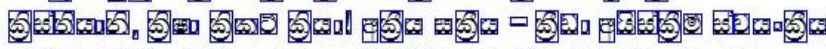

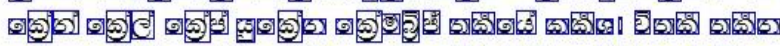

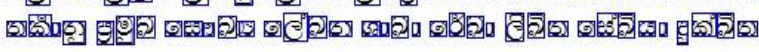

Fig. 9 Character segmentation

\begin{tabular}{|c|c|c|c|c|c|}
\hline \multicolumn{2}{|c|}{ Box Coordinates } & \multicolumn{2}{|c|}{ Box Data } & Box View & \multirow[b]{2}{*}{ Height } \\
\hline & Char & $\mathrm{x}$ & $Y$ & Width & \\
\hline 277 & से & 499 & 252 & 13 & 14 \\
\hline 278 & อ & 513 & 254 & 10 & 12 \\
\hline 279 & cs & 526 & 257 & 11 & 9 \\
\hline 280 & \% & 540 & 259 & 5 & 5 \\
\hline 281 & 2018 & 547 & 252 & 15 & 19 \\
\hline 282 & cs & 564 & 257 & 11 & 9 \\
\hline
\end{tabular}

Fig. 10 Sample of box information 


\section{Training the model}

The training was performed as an iterative process until better results were obtained. Firstly, the training models were done for individual data sets of computer-generated images for given font types and sizes. Secondly, we combined the training data sets for multiple fonts and multiple sizes and trained the models. Thirdly, the training was performed using the scanned images and trained multiple models for the given font types and sizes. Finally, all the data sets of computer-generated images and scanned images were combined in several ways and trained multiple models.

\section{- Preparation Tesseract 4.0 version}

\section{A. Setting up the OCR Engine}

For setting up the Tesseract 4.0 version we selected Ubuntu environment. Since Tesseract 4.0 deals with deep learning techniques such as Long Short-Term Memory (LSTM), the Ubuntu operating system provides full compatibility for OCR engines. And all the tasks were carried out in the terminal and instructions were given as commands.

\section{B. Preparation of training data sets}

Training data plays an important role in Tesseract version 4.0. With the integration of deep learning techniques, more training data will result in good outcomes. For our experiment, we have employed 3 datasets which are available for the Sinhala language. Further details of the 1) UCSC 10 million Sinhala dataset, 2) common crawler Sinhala dataset and 3) Google dataset will be discussed in the next few lines.

1) UCSC 10 Million Word Sinhala Corpus: UCSC $10 \mathrm{M}$ Word Sinhala Corpus has been compiled by the Language Technology Research Laboratory - University of Colombo School of Computing (UCSC) in Sri Lanka. This text corpus contains a huge variety of Sinhala books including novels, short stories, translations, critiques written by renowned Sinhala writers, and Sinhala newspapers: Silumina, Dinamina, Lankadeepa and Lakbima. The UCSC 10 million dataset includes texts which belong to different eras in Sri Lanka. It also contains texts from various sources; the text is rich with different writings. Noise data and other textual data with different languages have been removed from this dataset in order to minimize the errors.

2) 5million+ sentences in Sinhala common crawler: In 2019, Guzman [16] presented two monolingual corpora for Sinhala. Those were a combination of $155 \mathrm{k}+$ sentences of filtered Sinhala Wikipedia and 5178k+ sentences of Sinhala common crawl. Since this study considered only textual data available online, the diversity of textual representation is considerably low. Furthermore, a high noise rate exists in this dataset with other common issues like the zero width joiner problem and the combination of multiple language textual data with Sinhala textual data. And these affect the overall accuracy of the system.

3) Google dataset for Sinhala is especially built with the Tesseract. This dataset includes variety of textual representations gathered in recent years.

4) UCSC $400 K$ distinct wordlist: This list of monolingual vocabulary was developed from the UCSC 10 million words Sinhala corpus by the Language Technology Research Lab of UCSC. The list includes 440,021 distinct entries and is available on the web.

After comparing these 3 datasets, the UCSC 10 million Sinhala dataset [17] was selected by the authors due to the enrichment of textual combinations in different eras and less noise data. UCSC 400K Distinct Word List [18] was also combined with the existing Tesseract word list.

As a special feature, the Tesseract version 4.0 generates the tiff file and box file automatically. Additionally, image and corresponding UTF-8 text transcription are generated on 1stmf file at the process of font training. Also in Tesseract 4.0 the clustering steps (mftraining, cntraining, shape clustering) are replaced with a single slow lstm training step.

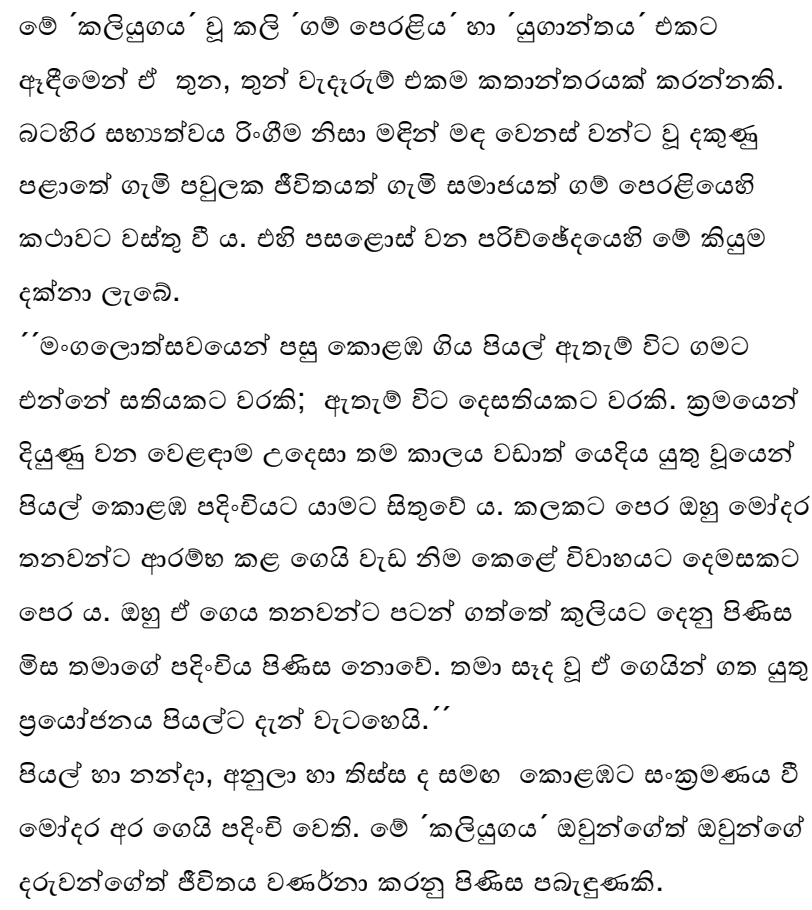

Fig. 11 Sample of training data for Tesseract 4.0

\section{Selection of font types and sizes}

Since typefaces are significant in training an OCR system, we investigated the commonly used Sinhala fonts to train the OCR model in Tesseract 4.0. Though there are hundreds of non-Unicode fonts available for the Sinhala script, they have no unique character code point for identification. Owing to its 16-bit encoding, UNICODE is theoretically able to support over 65,000 unique character code points [19] and we selected 9 Unicode fonts from the limited number of Sinhala Unicode fonts available. They include Unicode fonts which are most commonly used in printed and digital media [20]. The font types involved with the research is given below.

$\begin{array}{cl}\circ & \text { Noto-Sans font } \\ \circ & \text { LKLug font } \\ \circ & \text { Malithi font } \\ \circ & \text { Dinamina font } \\ \circ & \text { Iskolapotha font } \\ \circ & \text { BhashitaComplex font }\end{array}$




\section{Training the model}

As pre-processing steps for noise removal, adaptive thresholding, page layout analysis and connected component analysis were performed by the Tesseract OCR engine. The following steps were followed to train the model. Initially generated training data is provided as the input to the engine and extract the generated model. Then the model was fine tuned to decrease the error rate and finally the fine-tuned model was combined with the initial trained model. We combine multiple fonts for model creation. Single font models, Double font models and Triple font models were used for analysis.

\section{EVALUATION AND RESULTS}

For the evaluation process, we considered both Tesseract 3.0 and Tesseract 4.0. As the first phase, the Tesseract version 3.0 was evaluated by character level. Meanwhile, the Tesseract 4.0 was evaluated at both character and word level. The developed OCR models have been tested with 30 images selected for three different categories (10 for each category). When selecting images for testing we chose non identical images with different typefaces and different image qualities.

1) Old Sinhala newspapers: The testing data for this category was selected from archived images of Sinhala newspapers published in $1870-1890$. The newspapers

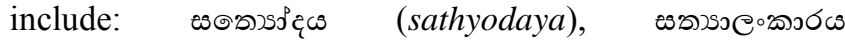

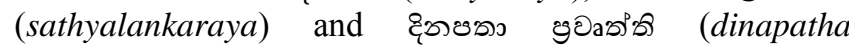
prawruththi). All the images are in 200 DPI.

2) Old Sinhala books: Testing images for this category were selected from old Sinhala books which are printed on Letterpress printing. The old books selected include:

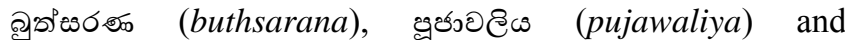

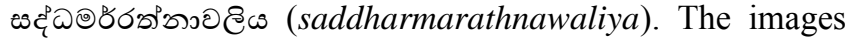
in this category are in 72 DPI.

3) Contemporary Sinhala books: The books printed with computerized fonts were selected for this category. 10 images of randomly selected pages from 10 books were taken and they were scanned for 300 DPI.

To calculate the accuracy of the systems we compare the common and different characters between original and OCR document.

Accuracy $(\%)=\quad$ Count of common characters $\quad X \quad 100 \%$ Count of (common characters + different characters)

\section{A. Evaluation of the models from Tesseract 3.0}

The evaluation of Tesseract version 3.0 was conducted only for the third category of testing images for two reasons. Firstly, the results for the other two categories were not at satisfying level and secondly, we gave our main priority for the evaluation of Tesseract version 4.0 Therefore, we selected the most accurate model (Scanned-iskolapotha model) out of 18 multiple models created by varying different font types and sizes. Original data of the testing samples consist of 2592 words and 16380 characters. Testing results are illustrated in table i.
TABLE I

TESSERACT 3.0 RESULTS OF OCR DOCUMENTS

\begin{tabular}{|l|l|l|l|}
\hline Font type & $\begin{array}{c}\text { Recognized } \\
\text { character } \\
\text { count }\end{array}$ & $\begin{array}{l}\text { Recognized } \\
\text { word count }\end{array}$ & $\begin{array}{c}\text { Accuracy } \\
\text { of the } \\
\text { system }\end{array}$ \\
\hline Iskolapotha & 16962 & 2507 & $36.89 \%$ \\
\hline
\end{tabular}

\section{B. Evaluation of the models from Tesseract 4.0}

The models generated from Tesseract 4.0 OCR engine were evaluated for the three categories of testing samples explained above. From the generated models, all the models of individual fonts and three selected Combined Models (CM) were evaluated. The same set of testing images were used in the evaluation process.

For the first category of evaluation, we selected 10 images from old newspapers and they consist of 1557 words and 9821 characters. Some of the texts in these images are even hard to read by a human. The results for the first category of images are shown in table ii.

TABLE II

CATEGORY 01 RESULTS OF OCR DOCUMENTS

\begin{tabular}{|l|l|l|l|}
\hline \multicolumn{1}{|c|}{ Font type } & $\begin{array}{c}\text { Recognized } \\
\text { character } \\
\text { count }\end{array}$ & $\begin{array}{l}\text { Recognized } \\
\text { word count }\end{array}$ & $\begin{array}{c}\text { Accuracy } \\
\text { of the } \\
\text { system }\end{array}$ \\
\hline Noto-Sans & 10142 & 1462 & $61.43 \%$ \\
\hline LK-LUG & 10031 & 1441 & $61.66 \%$ \\
\hline Malithi & 10094 & 1516 & $65.51 \%$ \\
\hline Iskolapotha & 10067 & 1458 & $67.02 \%$ \\
\hline Dinamina & 9897 & 1426 & $59.83 \%$ \\
\hline $\begin{array}{l}\text { Bashitha } \\
\text { Noto-LKLug } \\
\text { (CM) }\end{array}$ & 10071 & 1451 & $61.96 \%$ \\
\hline $\begin{array}{l}\text { Malithi-Lug } \\
\text { (CM) }\end{array}$ & 10003 & 1449 & $61.51 \%$ \\
\hline $\begin{array}{l}\text { Noto-Lug- } \\
\text { Malithi }\end{array}$ & 10035 & 1445 & $64.40 \%$ \\
\hline
\end{tabular}

There were 3032 words and 18074 of characters in the images of category 2 , the old books printed in letterpress printing era. The results obtained are illustrated in table iii. 
TABLE III

CATEGORY 02 RESULTS OF OCR DOCUMENTS

\begin{tabular}{|l|l|l|l|}
\hline \multicolumn{1}{|c|}{ Font type } & $\begin{array}{c}\text { Recognized } \\
\text { character } \\
\text { count }\end{array}$ & $\begin{array}{l}\text { Recognized } \\
\text { word count }\end{array}$ & $\begin{array}{c}\text { Accuracy } \\
\text { of the } \\
\text { system }\end{array}$ \\
\hline Noto-Sans & 18623 & 3019 & $85.13 \%$ \\
\hline LK-LUG & 18584 & 3012 & $87.15 \%$ \\
\hline Malithi & 18774 & 3039 & $87.07 \%$ \\
\hline iskolapotha & 18688 & 3022 & $85.28 \%$ \\
\hline $\begin{array}{l}\text { Dinamina } \\
\text { Bashita }\end{array}$ & 18428 & 2807 & $84.97 \%$ \\
\hline $\begin{array}{l}\text { Noto-LKLug } \\
\text { (CM) }\end{array}$ & 18627 & 3023 & $87.53 \%$ \\
\hline $\begin{array}{l}\text { Malithi-Lug } \\
\text { (CM) }\end{array}$ & 18704 & 3017 & $86.06 \%$ \\
\hline $\begin{array}{l}\text { Noto-Lug- } \\
\text { Malithi }\end{array}$ & 18461 & 3010 & $87.52 \%$ \\
\hline
\end{tabular}

Third category of 10 images captured from contemporary Sinhala books and they consist 2592 words and 16151 characters. The results are denoted in table iv.

TABLE IV

CATEGORY 03 RESULTS OF OCR DOCUMENTS

\begin{tabular}{|l|l|l|l|}
\hline \multicolumn{1}{|c|}{ Font type } & $\begin{array}{c}\text { Recognized } \\
\text { character } \\
\text { count }\end{array}$ & $\begin{array}{l}\text { Recognized } \\
\text { word count }\end{array}$ & $\begin{array}{c}\text { Accuracy } \\
\text { of the } \\
\text { system }\end{array}$ \\
\hline Noto-Sans & 16476 & 2620 & $85.91 \%$ \\
\hline LK-LUG & 16306 & 2615 & $84.91 \%$ \\
\hline Malithi & 16530 & 2626 & $86.14 \%$ \\
\hline iskolapotha & 16391 & 2635 & $87.63 \%$ \\
\hline Dinamina & 16104 & 2459 & $85.49 \%$ \\
\hline Bashita & 16178 & 2591 & $87.49 \%$ \\
\hline $\begin{array}{l}\text { Noto-LKLug } \\
\text { (CM) }\end{array}$ & 16335 & 2627 & $84.83 \%$ \\
\hline $\begin{array}{l}\text { Malithi-Lug } \\
\text { (CM) }\end{array}$ & 16338 & 2796 & $85.49 \%$ \\
\hline $\begin{array}{l}\text { Noto-Lug- } \\
\text { Malithi(CM) }\end{array}$ & 16259 & 2613 & $86.59 \%$ \\
\hline
\end{tabular}

When three categories of input image sets were considered, old newspapers have a low accuracy rate in character recognition due to the high existence of noises and low quality of images. In the old Sinhala book category Malithi, LKLug and the combined model of Noto Sans, LKLug and Malithi were given best accuracy when recognizing characters. The model trained with the font Iskolapotha obtained the highest accuracy rate in $3^{\text {rd }}$ category of contemporary Sinhala books. When analysing OCR outputs, some identifiable improvement can be done in the recognition process of the system.

Moreover, as another part of this research we randomly selected 5 images from the contemporary Sinhala books and converted for low DPI count (from 300px to 96px). Thereafter, we chose our best 3 models in the contemporary Sinhala books category and evaluated the performance. A total count of 1482 words and 8735 characters were there in the selected 5 image data.

TABLE V

COMPARISON WITH THE LOW DPI LEVEL

\begin{tabular}{|l|l|l|l|}
\hline Font type & $\begin{array}{c}\text { Recognized } \\
\text { character } \\
\text { count }\end{array}$ & $\begin{array}{c}\text { Recognized } \\
\text { word count }\end{array}$ & $\begin{array}{c}\text { Accuracy } \\
\text { of the } \\
\text { system }\end{array}$ \\
\hline iskolapotha & 8983 & 1481 & $87.88 \%$ \\
\hline Malithi & 9067 & 1479 & $86.09 \%$ \\
\hline $\begin{array}{l}\text { Noto-Lug- } \\
\text { Malithi } \\
\text { (CM) }\end{array}$ & 8895 & 1473 & $87.40 \%$ \\
\hline
\end{tabular}

As a special note, after reducing DPI count, some character combinations which were not recognized correctly but samples with 300 DPI were able to recognized. For instance yanshaya was not recognized well in previous efforts but with this modification yanshaya was recognized well. Some clearly identifiable errors in the recognition process of our OCR system has been briefly noted below.

- Confusing with similar shaped individual characters. (e.g.

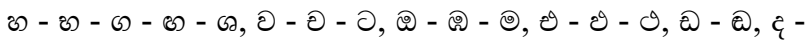

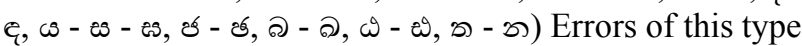
frequently occur in 1 st and 2 nd categories of testing images. When the images are not clear, the tiny variations of the characters are difficult to be captured.

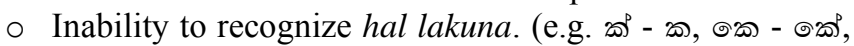
อ - อ, ๑อ - ๑อి)

- Confusing with vowel modifiers and hal lakuna in the same character or in different characters. (e.g. $\delta$ - $\delta$ - $\delta$ -

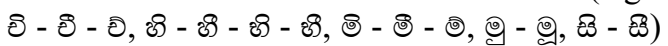

- Misidentification of rakaranshaya with papilla, the vowel

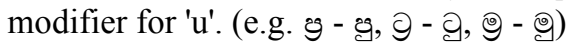

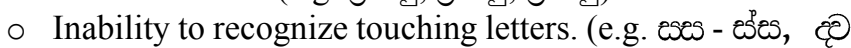

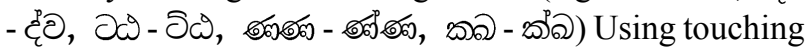
letters was a writing style in old Sinhala. And in Pali it is a rule, as Pali does not have a sign like hal lakuna to show the absence of the inherent vowel. This writing style can occur in some testing images of category 1 and 2 . Since 
contemporary writing does not follow this style, the training data is not rich with these sequences. This has resulted in not recognizing touching letters.

- Inability to recognize compound consonants. The compound consonants given in figure 6 hardly occur in contemporary Sinhala and therefore they are not well recognized.

Some English characters were also in the testing images of all three categories. As we focused on developing a better recognition model for Sinhala characters, we did not include enough English text data in the training process, this resulted in some errors in recognition and affected the overall accuracy of the system. However, the above limitations will be considered in the next stage as a future enhancement.

\section{CONCLUSION AND FUTURE WORKS}

In this paper we presented a process of developing an Optical Character Recognition system for Sinhala. In this research we identified the characteristics of Sinhala script along with properties of writing style in Sinhala scripts. The training process of the OCR model was initiated with Tesseract 3.0 and later moved to Tesseract 4.0, as it was the state of the art of deep learning.

The evaluation was carried out by comparing the results with the different types of Sinhala fonts and adapting systems to recognize varieties of test data gathered from different sources. Although we tested some samples with the model built from a Sinhala common crawl dataset, overall accuracy is less compared to others and unable to identify characters.

According to the results, our system model trained with font iskolapotha gave accuracy of $87.63 \%$ in contemporary Sinhala books. In the Sinhala old book category, models developed using fonts Malithi, LKLug and combined font models using Noto Sans, LKLug and Malithi gave accuracies of $87.07 \%, 87.15 \%$ and $87.52 \%$ respectively. Meanwhile in the old Sinhala newspaper category $67.02 \%$ of accuracy was obtained from the model developed with font iskolapotha.

Developing OCR systems for low resource languages needed a considerable amount of effort from both linguistics and computer science domain areas. Analysing linguistics rules and mapping them with computer science is quite challenging for low resources languages like Sinhala and Tamil. In this stage of the research, we focused highly, only on the recognition of the Sinhala script. As mentioned in the above sections, the Sinhala script is also used to write "Pali" and "Sanskrit" languages in Sri Lanka. As a future enhancement we will work on identifying touching and conjoining letters which occur frequently in Pali. We also plan to apply some n-gram or word embedding based postprocessing techniques to enhance the accuracy. Also in real world OCR can be categorized as one of sequence learning tasks. Therefore, it is necessary to predict the sequence of labels from noisy, unsegmented input data. As future work, we plan to combine connectionist temporal classification (CTC) with deep learning algorithms to train the Recurrent Neural Network (RNN) to label unsegmented sequences directly. Moreover, neural net compressions and conventional neural machine translations for Sinhala OCR will be studied in the future.

\section{ACKNOWLEDGMENT}

This work was carried out as a part of a project funded by Theekshana - Research and Development Company. We acknowledge Mrs. Dinuji Godigamuwa for assisting the work and thank all the members of Language Technology Research Laboratory of the University of Colombo School of Computing, Sri Lanka, who helped in various ways to make this work bear fruit.

\section{REFERENCES}

[1] R. Weerasinghe, A. Wasala, D. Herath, and V. Welgama, "Nlp applications of sinhala: Tts \& ocr," in Proceedings of the Third International Joint Conference on Natural Language Processing: Volume-II, 2008.

[2] Smith, R. (2007) 'An Overview of the Tesseract OCR Engine', in Ninth International Conference on Document Analysis and Recognition (ICDAR 2007), pp. 629-633. doi: 10.1109/ICDAR.2007.4376991.

[3] A. R. Weerasinghe, D. L. Herath, and N. P. K. Medagoda, "A nearestneighbor based algorithm for printed sinhala character recognition," Innov. a Knowl. Econ., p. 11, 2006.

[4] M. Rimas, R. P. Thilakumara, and P. Koswatta, "Optical character recognition for Sinhala language,” in 2013 IEEE Global Humanitarian Technology Conference: South Asia Satellite (GHTC-SAS), 2013, pp. $149-153$

[5] S. Ajward, N. Jayasundara, S. Madushika, and R. Ragel, "Converting printed Sinhala documents to formatted editable text," in 2010 Fifth International Conference on Information and Automation for Sustainability, 2010, pp. 138-143.

[6] H. W. H. Premachandra, C. Premachandra, T. Kimura, and H. Kawanaka, "Artificial neural network based sinhala character recognition," in International Conference on Computer Vision and Graphics, 2016, pp. 594-603.

[7] [Online]. Available: http://192.248.22.122/ocrsinhala/

[8] I. Anuradha, C. Liyanage, H. Wijayawardhana, and R. Weerasinghe, "Deep Learning Based Sinhala Optical Character Recognition (OCR)," in 2020 20th International Conference on Advances in ICT for Emerging Regions (ICTer), 2020, pp. 298-299.

[9] U. Manisha and S. R. Liyanage, "Sinhala Character Recognition using Tesseract OCR,” 2018.

[10] C. Liyanage, T. Nadungodage, and R. Weerasinghe, "Developing a commercial grade Tamil OCR for recognizing font and size independent text," in 2015 Fifteenth International Conference on Advances in ICT for Emerging Regions (ICTer), 2015, pp. 130-134.

[11] N. Mishra, C. Patvardhan, C. V. Lakshmi, and S. Singh, "Shirorekha chopping integrated tesseract ocr engine for enhanced hindi language recognition,” Int. J. Comput. Appl., vol. 39, no. 6, pp. 19-23, 2012.

[12] M. T. Chowdhury, M. S. Islam, B. H. Bipul, and M. K. Rhaman, "Implementation of an Optical Character Reader (OCR) for Bengali language," in 2015 International Conference on Data and Software Engineering (ICoDSE), 2015, pp. 126-131.

[13] S. Hussain, A. Niazi, U. Anjum, F. Irfan, and others, "Adapting Tesseract for complex scripts: an example for Urdu Nastalique," in 2014 11th IAPR International Workshop on Document Analysis Systems, 2014, pp. 191-195.

[14] R. M. Joshi and C. McBride, Handbook of Literacy in Akshara Orthography, vol. 17. Springer, 2019.

[15] J. W. Gair and W. S. Karunatilaka, "Literary Sinhala Inflected Forms: A Synopsis with a Transliteration Guide to Sinhala Script.," 1976.

[16] Guzmán, F., Chen, P. J., Ott, M., Pino, J., Lample, G., Koehn, P., ... \& Ranzato, M. (2019). Two new evaluation datasets for low-resource machine translation: Nepali-english and sinhala-english. arXiv 2019. arXiv preprint arXiv:1902.01382.

[17] [Online]. Available: http://ltrl.ucsc.lk/tools-and-resourses/

[18] [Online]. Available: http://trl.ucsc.lk/tools-and-resourses/

[19] V. K. Samaranayake, S. T. Nandasara, J. B. Disanayaka, A. R. Weerasinghe, and H. Wijayawardhana, "An introduction to UNICODE for Sinhala characters," Univ. Colombo Sch. Comput., 2003.

[20] R. Subasinghe, S. Eramudugolla, S. Samarawickrama and G. Dias, "Atomic Vs Anatomic Features of Sinhala Fonts", 10th Typography Meeting, 2019. 
APPENDIX A

Following include the images of three categories used for testing each OCR model.

ตรด8 O

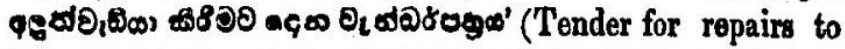

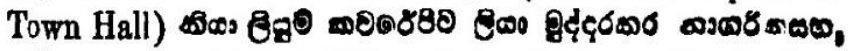

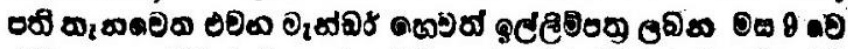

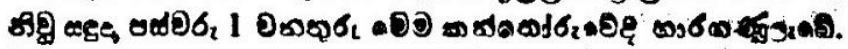

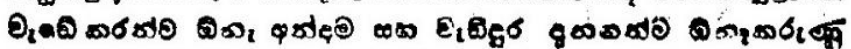

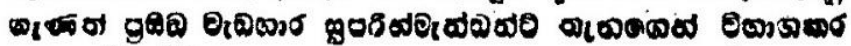

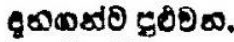

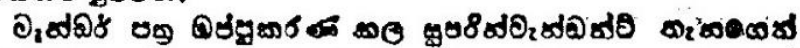

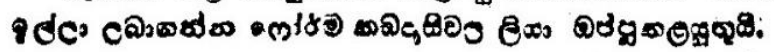

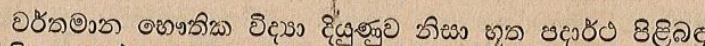

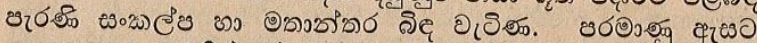

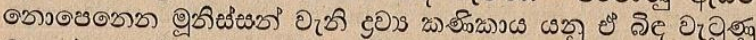

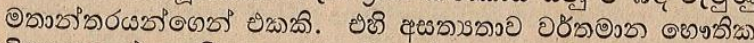

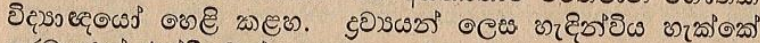

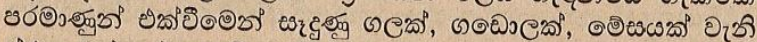

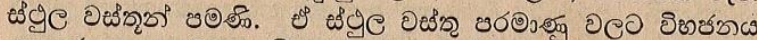

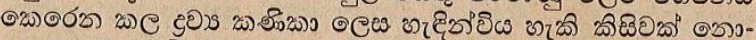

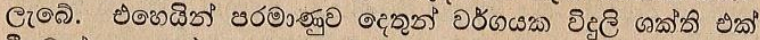

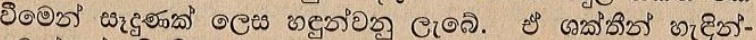

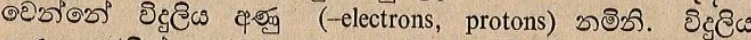

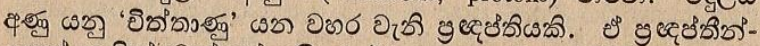

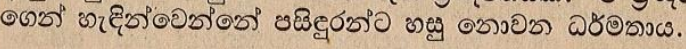

Fig A.3. A sample image of category 1 (contemporary Sinhala books)

Fig A.1. A sample image of category 1 (old Sinhala newspapers)

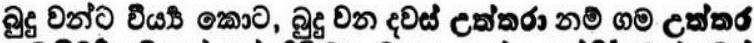

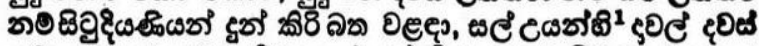

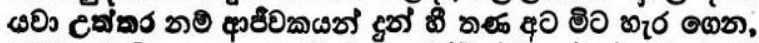

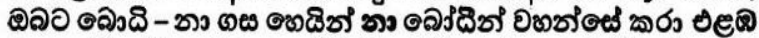

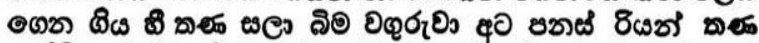

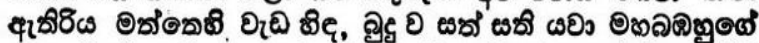

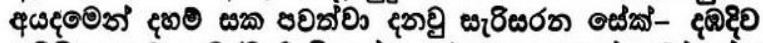

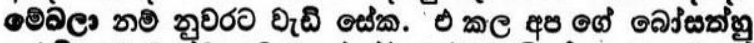

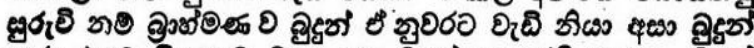

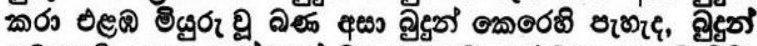

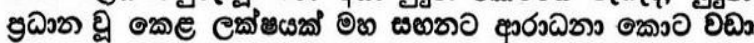

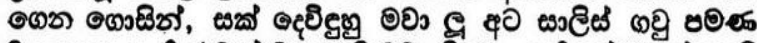

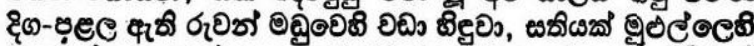

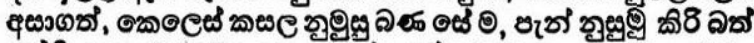

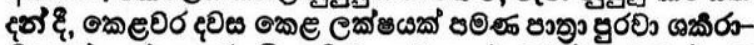

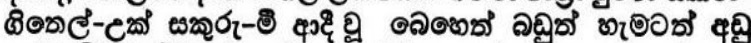

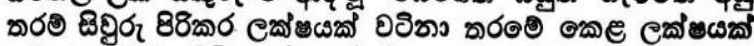

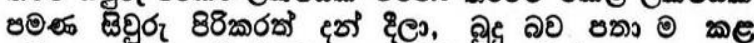

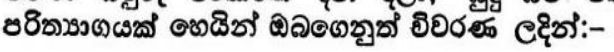

Fig A.2. A sample image of category 2 (old Sinhala books)

\section{APPENDIX B}

Interface of developed OCR system is shown in Figure B.

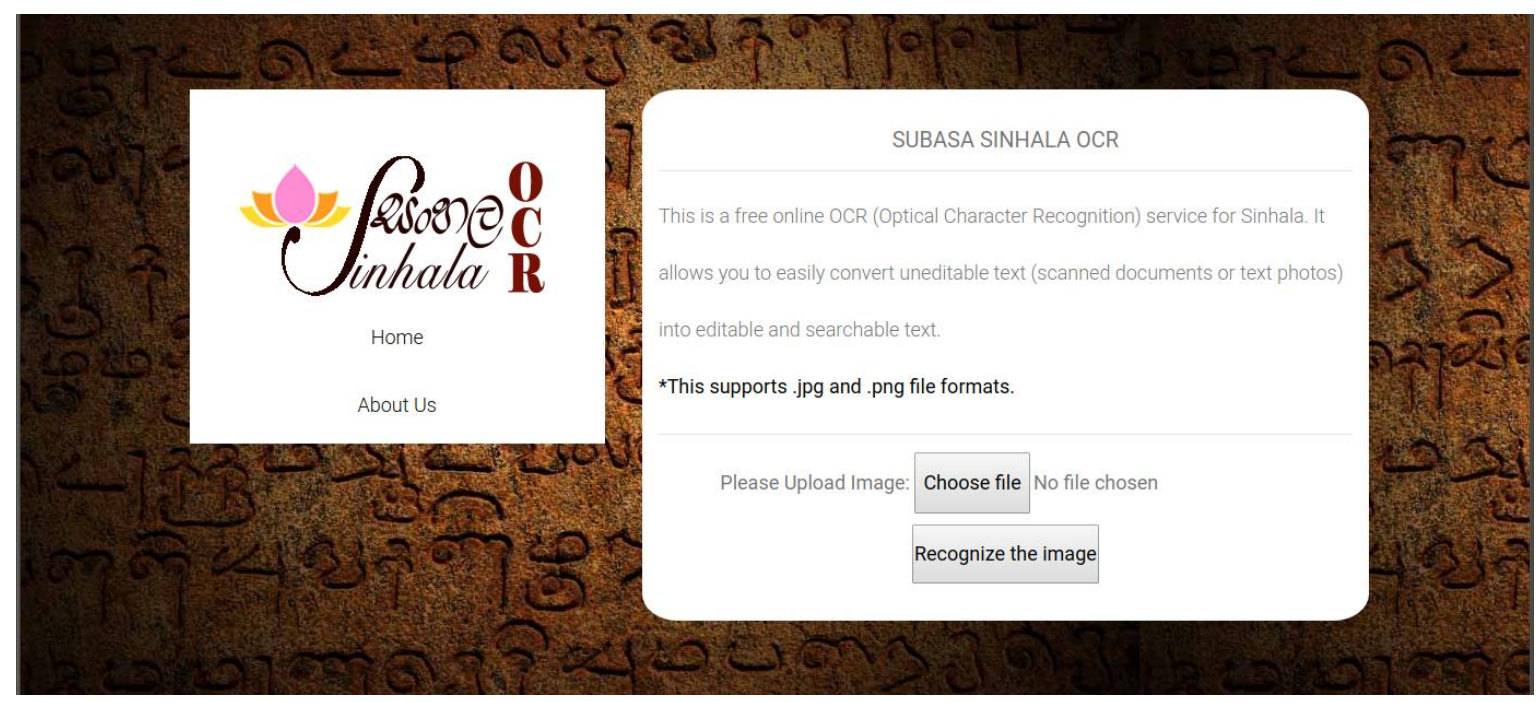

Fig B. Online Application developed for the Sinhala OCR 